\title{
A nonexistence result for CMC surfaces in hyperbolic 3-manifolds
}

\author{
William H. Meeks III ～Alvaro K. Ramos*
}

July 10,2020

\begin{abstract}
We prove that a complete hyperbolic 3-manifold of finite volume does not admit a properly embedded noncompact surface of finite topology with constant mean curvature greater than or equal to 1 .

Mathematics Subject Classification: Primary 53A10, Secondary 49Q05, $53 \mathrm{C} 42$.

Key words and phrases: Constant mean curvature, hyperbolic 3-manifolds, Calabi-Yau problem.
\end{abstract}

\section{Introduction.}

We continue the study of properly immersed surfaces of constant mean curvature $H$ in hyperbolic 3-manifolds of finite volume that began with the works of Collin, Hauswirth, Mazet and Rosenberg [2, 4] in the minimal case, and was extended to the $H \in(0,1)$ case by the authors [6].

In this paper we prove:

Theorem 1.1. A complete hyperbolic 3-manifold of finite volume does not admit a properly embedded noncompact surface of finite topology with constant mean curvature $H \geq 1$.

*The authors were partially supported by CNPq - Brazil, grant no. 400966/2014-0. 
Theorem 1.1 contrasts with [6, Proposition 4.8], where it is shown that, for any $H \geq 1$ and any noncompact hyperbolic 3-manifold $N$ of finite volume, there exists a complete, properly immersed annulus with constant mean curvature $H$. Therefore, the hypothesis of embeddedness in Theorem 1.1 is necessary. Moreover, in [1], together with Adams, we proved that for any $H \in[0,1)$ and any surface $S$ of finite negative Euler characteristic, there exists a hyperbolic 3-manifold of finite volume with a proper embedding of $S$ with constant mean curvature $H$. Furthermore, there are examples of closed surfaces in hyperbolic 3-manifolds of finite volume for any $H \geq 1$; namely geodesic spheres and tori and Klein bottles in its cusp ends.

The work of the first author with Tinaglia [7] allows one to replace the hypothesis of properness in Theorem 1.1 by the weaker assumption of completeness, since [7] shows that any complete, embedded, finite topology surface of constant mean curvature $H \geq 1$ in a complete hyperbolic 3-manifold is proper. Finally, there remains the question of whether or not there exist properly embedded surfaces of infinite topology and constant mean curvature $H \geq 1$ in hyperbolic 3manifolds of finite volume.

\section{Proof of Theorem 1.1.}

Theorem 1.1 follows directly from next lemma.

Lemma 2.1. A complete hyperbolic 3-manifold of finite volume $N$ does not admit a proper embedding of $A=\mathbb{S}^{1} \times[0, \infty)$ with constant mean curvature $H \geq 1$.

Proof. After passing to the oriented two-sheeted cover of $N$, we may assume without loss of generality that $N$ is orientable.

Arguing by contradiction suppose that $E \subset N$ is the image of a proper embedding of $A$ as stated in the lemma. Since $E$ is proper and $N$ is an orientable hyperbolic 3-manifold of finite volume, there exists some cusp end $\mathcal{C}$ of $N$ with the following properties:

1. $\partial E \cap \mathcal{C}=\emptyset$.

2. $\partial \mathcal{C}$ is a flat torus $\mathcal{T}(0)$ which intersects $E$ transversely in a finite collection of pairwise disjoint simple closed curves.

3. $E \cap \mathcal{C}$ contains a unique noncompact component $\Delta$, which is a planar domain. 
Since $\Delta$ is connected and $\partial \Delta$ separates $\partial E$ from the end of $E$, it follows that $\partial \Delta \subset \mathcal{T}(0)$ contains a unique simple closed curve $\gamma \subset \partial \Delta$ which generates the first homology group $H_{1}(E)$. Moreover, any other boundary component of $\partial \Delta$ is homotopically trivial in $E$, and hence, homotopically trivial in $N$. In particular, $i_{*}\left(\pi_{1}(\Delta)\right)$ is either trivial or an infinite cyclic subgroup of $\pi_{1}(\mathcal{C})$, where $i: \Delta \rightarrow \mathcal{C}$ is the inclusion map and $i_{*}: \pi_{1}(\Delta) \rightarrow \pi_{1}(\mathcal{C})$ is the induced map on fundamental groups, after choosing a base point on $\gamma$.

We let $\Pi: \mathbb{H}^{3} \rightarrow N$ be the universal cover of $N$ and let $B \subset \mathbb{H}^{3}$ be a horoball such that $\left.\Pi\right|_{B}: B \rightarrow \mathcal{C}$ is the universal cover of $\mathcal{C}$. Using the half-space model for $\mathbb{H}^{3}$, we assume, without loss of generality, that $B$ is the region $B=\{(x, y, z) \in$ $\left.\mathbb{H}^{3} \mid z \geq 1\right\}$.

Case 2.2. $i_{*}: \pi_{1}(\Delta) \rightarrow \pi_{1}(\mathcal{C})$ is trivial.

Since $i_{*}$ is trivial, $i: \Delta \rightarrow \mathcal{C}$ admits a lift $\widetilde{i}: \Delta \rightarrow B$, whose image $\widetilde{\Delta}$ is a properly embedded planar domain in $B$ with $\partial \widetilde{\Delta} \subset \partial B$. By [3, Theorem 10] (for $H=1$ ) and [5, Theorem 6.9] (for $H>1$ ), it follows that $\widetilde{\Delta}$ is asymptotic to a constant mean curvature $H$ annulus $\mathcal{A} \subset B$, in the sense that a subend of $\widetilde{\Delta}$ is a graph in exponential normal coordinates over a subend of $\mathcal{A}$ with graphing function converging in the $C^{2}$-norm to zero for any divergent sequence of points. Moreover, $\mathcal{A}$ admits a vertical axis of rotational symmetry, and there are three possibilities:

1. $H=1$ and $\mathcal{A}$ is the end of a horosphere;

2. $H=1$ and $\mathcal{A}$ is the end of an embedded catenoid cousin;

3. $H>1$ and $\mathcal{A}$ is the end of a Delaunay surface.

Note that the Catenoid cousin in item 2 is embedded because any end representative of a nonembedded Catenoid cousin is never contained in a horoball.

In each case, $\mathcal{A}$ has bounded norm on its second fundamental form and infinite area; hence, since $\widetilde{\Delta}$ is asymptotic to $\mathcal{A}$ in the $C^{2}$-norm, it follows that $\widetilde{\Delta}$ also has bounded norm of its second fundamental form $\left\|A_{\widetilde{\Delta}}\right\|$ and infinite area. Next, we use these properties to get a contradiction.

Since $\widetilde{\Delta}$ is a complete, properly embedded surface with $\partial \widetilde{\Delta} \subset \partial B$, then $\widetilde{\Delta}$ defines a mean convex region $M \subset B$ with $\partial M \backslash \partial B=\widetilde{\Delta}$. Moreover, since $\mathbb{H}^{3}$ is a homogeneously regular manifold and $\widetilde{\Delta}$ has compact boundary and separates $B$, the bound on $\left\|A_{\widetilde{\Delta}}\right\|$ gives the existence of a one-sided regular neighborhood in $M$ of radius $\delta>0$, see [8, Lemma 3.1]. Since $\widetilde{\Delta}$ has infinite area and $\delta>0$, then $M$ has infinite volume. 


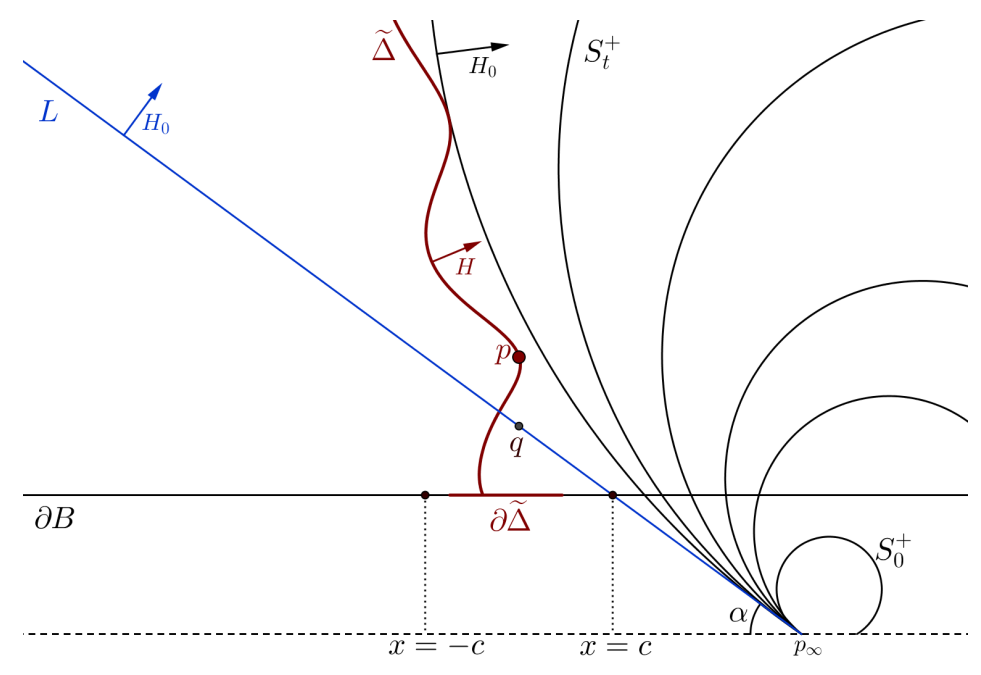

Figure 1: $\widetilde{\Delta}$ has constant mean curvature $H \geq 1$ and each hypersphere $S_{t}^{+}$has constant mean curvature $H_{0}=\cos (\alpha)$. The plane $L$ separates $p$ and $\partial \widetilde{\Delta}, p$ lies in the mean convex region of $\mathbb{H}^{3}$ determined by $L$ and $S_{t}^{+}$converge, when $t \rightarrow \infty$, to $L$.

Let $\sigma: B \rightarrow B$ be a parabolic translation of $\mathbb{H}^{3}$ that is a covering transformation of $\Pi$. Since $\Delta$ is embedded, then $\sigma(\widetilde{\Delta}) \cap \widetilde{\Delta}=\emptyset$; thus, either $\sigma(M) \cap M=\emptyset$ or $\sigma(M) \subset M$. Since $\sigma$ is a translation, the latter is not possible and we obtain that $\sigma(M) \cap M=\emptyset$. Hence, $\left.\Pi\right|_{M}: M \rightarrow \mathcal{C}$ is injective, which is a contradiction because $\mathcal{C}$ has finite volume, and this proves Lemma 2.1 when $i_{*}$ is trivial.

Case 2.3. $i_{*}: \pi_{1}(\Delta) \rightarrow \pi_{1}(\mathcal{C})$ is nontrivial.

In this case, $i_{*}\left(\pi_{1}(\Delta)\right)$ is a $\mathbb{Z}$-subgroup of $\mathbb{Z} \times \mathbb{Z}=\pi_{1}(\mathcal{C})$, generated by $i_{*}([\gamma])$. Let $\widetilde{\Delta} \subset B$ be a connected component of $\Pi^{-1}(\Delta)$. Then $\widetilde{\Delta}$ is a complete, noncompact, properly embedded planar domain in $B$ with $\partial \widetilde{\Delta} \subset \partial B$; in particular, it defines a mean convex region $M \subset B$ with $\partial M \backslash \partial B=\widetilde{\Delta}$.

Also, note that $\widetilde{\Delta}$ is invariant under the parabolic covering transformation $\theta: B \rightarrow B$ corresponding to $i_{*}([\gamma]) \in \pi_{1}(\mathcal{C})$. Since $\partial \Delta$ is compact, $\partial \widetilde{\Delta}$ stays a finite distance $c>0$ from a line $l \subset \partial B$, invariant under $\theta$. In order to clarify the next argument, we apply a rotation around the $z$-axis of $\mathbb{H}^{3}$ to assume that $l=\{(0, y, 1) \mid y \in \mathbb{R}\}$; hence, $\partial \widetilde{\Delta} \subset\{(x, y, 1) \mid x \in(-c, c), y \in \mathbb{R}\}$. Also, after possibly reflecting through the $x z$-plane, we may assume that $\{(x, y, 1) \mid x \geq$ 
c) $\subset \partial M$. See Figure 1 .

Let $p=\left(x_{1}, y_{1}, z_{1}\right) \in \widetilde{\Delta}$ be such that $z_{1}>1$ and $x_{1} \in(-c, c)$. Let $q=$ $\left(x_{1}, y_{1}, \frac{z_{1}+1}{2}\right)$ and let $L$ be the tilted plane through $q$ containing the line $\{(c, y, 1) \mid$ $y \in \mathbb{R}\}$. Then, $L$ is an equidistant surface to a totally geodesic vertical plane and, when oriented with respect to the upper normal vector field, has constant mean curvature $H_{0}=\cos (\alpha) \in(0,1)$, where $\alpha$ is the acute, Euclidean angle between $L$ and $\{z=0\}$. Note that $L$ separates $\partial \widetilde{\Delta}$ and $p$, and that $p$ lies in the mean convex region $U$ defined by $L$ in $\mathbb{H}^{3}$.

Let $S_{0}$ be a totally geodesic surface of $\mathbb{H}^{3}$ such that $S_{0} \subset U$, with asymptotic boundary meeting the asymptotic boundary of $L$ in a single point. Let $S_{0}^{+}$denote the equidistant surface to $S_{0}$ with constant mean curvature $H_{0}$ with respect to the inner orientation. Note that we may choose $S_{0}$ so that $S_{0}^{+} \cap \partial B=\emptyset$, as shown in Figure 1.

Let $M^{+} \subset \mathbb{H}^{3}$ denote the mean convex region defined by $S_{0}^{+}$. Then, there is a product foliation $\left\{S_{t}^{+}\right\}_{t \geq 0}$ of $U \backslash M^{+}$such that each surface $S_{t}^{+}$is equidistant to a totally geodesic surface of $\mathbb{H}^{3}$ and has constant mean curvature $H_{0}$ with respect to the inward orientation; when $t \rightarrow \infty$, the surfaces $S_{t}^{+}$converge to $L$.

Since $p \in \widetilde{\Delta} \subset B$, then $p \notin M^{+}$. Then, the fact that $p \in U$ implies that $\left(\cup_{t \geq 0} S_{t}^{+}\right) \cap \widetilde{\Delta} \neq \emptyset$. But because $S_{t}^{+} \cap B$ is compact for all $t \geq 0$, there exists a smallest $T>0$ such that $S_{T}^{+} \cap \widetilde{\Delta} \neq \emptyset$. Since our construction gives that $\partial \widetilde{\Delta} \cap U=\emptyset$, any point $w$ in $S_{T}^{+} \cap \widetilde{\Delta}$ is interior to both $\widetilde{\Delta}$ and $S_{T}^{+}$, and then $S_{T}^{+}$ and $\widetilde{\Delta}$ intersect tangentially at $w$. Finally, since $S_{T}^{+} \cap B \subset M$, the mean curvature comparison principle implies that $H_{0} \geq H$, which is a contradiction, and this proves Lemma 2.1.

\section{References}

[1] C. Adams, W. H. Meeks III, and A. Ramos. Totally umbilical surfaces in hyperbolic 3-manifolds. Preprint at https://arxiv.org/pdf/2007.03166.pdf.

[2] P. Collin, L. Hauswirth, L. Mazet, and H. Rosenberg. Minimal surfaces in finite volume non compact hyperbolic 3-manifolds. Ann. of Math. (2), 145$1: 1-31,1997$.

[3] P. Collin, L. Hauswirth, and H. Rosenberg. The geometry of finite topology Bryant surfaces. Ann. of Math., 153(3):623-659, 2001. MR1836284 (2002j:53012), Zbl 1066.53019. 
[4] P. Collin, L. Hauswirth, and H. Rosenberg. Minimal surfaces in finite volume hyperbolic 3-manifolds and in $M \times S, M$ a finite area hyperbolic surface. Amer. J. Math., 140(4):1075-1112, 2018. DOI 10.1353/ajm.2018.0024.

[5] N. Korevaar, R. Kusner, W. H. Meeks III, and B. Solomon. Constant mean curvature surfaces in hyperbolic space. American J. of Math., 114:1-43, 1992. MR1147718, Zbl 0757.53032.

[6] W. H. Meeks III and A. K. Ramos. Properly immersed surfaces in hyperbolic 3-manifolds. J. Differential Geom., 112(2):233-261, 2019. DOI $10.4310 / j d g / 1559786424$ https://doi.org/10.4310/jdg/1559786424, MR3960267.

[7] W. H. Meeks III and G. Tinaglia. Embedded Calabi-Yau problem in hyperbolic 3-manifolds. Work in progress.

[8] W. H. Meeks III and G. Tinaglia. Existence of regular neighborhoods for $H$-surfaces. Illinois J. of Math., 55(3):835-844, 2011. MR3069286, Zbl 1269.53014.

William H. Meeks, III at profmeeks@gmail.com Mathematics Department, University of Massachusetts, Amherst, MA 01003

Álvaro K. Ramos at alvaro.ramos@ufrgs.br Departmento de Matemática Pura e Aplicada, Universidade Federal do Rio Grande do Sul, Brazil 